\title{
Unmet Needs and Therapeutic Strategies in Cardio-Hemato-Oncology
}

\author{
Nili Ronit Schamroth Pravda Ran Kornowski \\ Cardiology Department, Rabin Medical Center, Petach Tikva, Israel; Sackler Faculty of Medicine, Tel Aviv University, \\ Tel Aviv, Israel
}

\author{
Keywords \\ Cardio-hemato-oncology - Cardiovascular complications . \\ Cardiotoxicity
}

\begin{abstract}
With the increasing survival of cancer patients and therapeutic advancements, the cardiovascular complications of therapy have become a significant issue. A dedicated, integrated team is important in providing the balance between acquiring a hematological/oncological response and cardiovascular morbidity and mortality. This article aims to shed light on this field - providing some examples of therapies with cardiotoxic potential as well as the cardiologist's approach to patient assessment before, during, and after therapy.
\end{abstract}

o 2018 S. Karger AG, Basel

\section{Introduction}

The advancement of cancer treatments in recent years has been tremendous. Not only has this led to improving overall survival but also to an increase in exposure to cancer therapies and their various side effects. One of the prominent effects is on the cardiovascular system, and the cardiotoxic effects of chemotherapy and radiation have been described in the literature since 1967 [1]. The cardiovascular complications of these therapies extend over

\section{KARGER}

() 2018 S. Karger AG, Basel

E-Mail karger@karger.com

www.karger.com/aha all spheres of cardiology - myocardial dysfunction, accelerated coronary artery disease, increased arrhythmic potential, etc. These complications can manifest both early, during initial therapy, but also with late manifestations years after initial remission. These effects are both directly due to therapeutic agents as well as due to the indirect effects of accelerated heart disease in patients with conventional underlying risk factors, heart disease, and various comorbidities.

The field of cardio-hemato-oncology is still in its infancy and, unfortunately, often underprovided and underutilized [2]. The need for an integrated team and multidisciplinary approach to these patients is vital to minimize the potential morbidity and mortality of this fragile population. These patients are complex, often elderly with various comorbidities, suffering from malignancies which themselves can cause cardiovascular complications, such as hyperviscosity and increased thrombotic risk, as well as receiving potentially cardiotoxic therapies.

This article aims to highlight the important role of the cardio-hemato-oncology team - in screening, treating, and monitoring these patients. This field is immense and so this article will specifically focus on the cardiovascular burden of 3 commonly used therapies in hematology to highlight the cardio-hemato-oncology field - namely the tyrosine kinase inhibitors (TKIs), the immunomodulatory drugs used in the treatment of multiple myeloma, and the long-term cardiovascular side effects of radiation

Dr. Nili Schamroth Pravd

Cardiology Department, Rabin Medical Center

39 Ze'ev Jabotinsky Street

Petach Tikva 4941492 (Israel)

E-Mail drnscham@gmail.com 
therapy. While the focus is on the therapies and side effect profiles, multiple factors need to be taken into consideration when managing these patients - the patients' baseline characteristics and comorbidities, the nature of the disease/diagnosis, concomitant medication, and of course the patients' requests.

\section{Therapies with Cardiotoxic Potential}

Drugs with cardiotoxic potential have classically been categorized into 2 groups: type I agents having a dosedependent cardiotoxicity that causes mainly irreversible ventricular dysfunction (e.g., anthracyclines) and type II agents that exhibit a cardiotoxicity that is not dose dependent and is mainly reversible (e.g., trastuzumab) [3]. However, with more awareness of the potential side effects and emphasis on screening for preclinical cardiac dysfunction, this division is slowly dissolving, and most drugs do not fit into clear categories.

\section{Tyrosine Kinase Inhibitors}

TKIs are the initial treatment of choice for long-term control of patients with chronic myeloid leukemia and have been a "game changer" in improving the life-expectancy of these patients. With the advent of newer smallmolecule TKIs, the broad range of indications has been extended. These drugs have been associated with an increased risk of multiple cardiovascular side effects. While imatinib, the original first-generation TKI, has a relatively low rate of cardiotoxicity, the second- and third-generation TKIs have multiple vascular side effects, including QT prolongation, pulmonary hypertension in patients treated with dasatinib, peripheral arterial occlusive disease in patients treated with nilotinib, and venous and arterial vascular occlusive events with ponatinib treatment [4]. A clear example of this was evident in the PACE trial that evaluated ponatinib. The trial halted enrolment of patients after a follow-up period of 24 months, when $11.8 \%$ of the patients had serious arterial thrombotic events, most commonly cardiovascular events [5]. Following this, the US Food and Drug Administration (FDA) placed a hazard warning on ponatinib and warned of the risk of arterial occlusion as well as heart failure [4, 6]. More recent data suggests that the cardiovascular side effects can affect up to $25 \%$ of patients [7]. While these more potent drugs improve the hematological response of patients, the cardiovascular side effects can be calami-

Unmet Needs and Therapeutic Strategies

in Cardio-Hemato-Oncology tous to the patients' overall wellbeing. Considering this high rate of arterial thrombosis, the value of prophylactic aspirin has been deliberated. Although there is no consensus, some subanalyses have shown aspirin prophylaxis to be beneficial at providing a lower incidence of atherothrombotic events in patients receiving TKIs [7]. It is vital that treating physicians are aware of these potential side effects, especially considering the need for long-term therapy of TKIs in these patients, often spanning years of therapy.

\section{Immunomodulatory Drugs}

Another drug group that has received a FDA hazard warning has been the immunomodulatory drugs (thalidomide, lenalidomide, and pomalidomide) used prominently in the treatment of multiple myeloma. These drugs are known historically for their teratogenic side effects, but also have a high rate of mainly venous but also arterial thromboembolic events. This risk is increased considerably when administered in conjunction with glucocorticosteroids (up to 26\%) as done in most treatment regimens [8]. It must be noted that patients with multiple myeloma have an increased risk of venous thromboembolism (VTE) not only because of therapies, but also due to the disease itself, hyperviscosity, and other traditional VTE risk factors. Due to this significant thrombotic risk, the International Myeloma Working Group has recommended the routine use of VTE prophylaxis for patients under immunomodulatory drugs - aspirin for low-risk patients and anticoagulation for high-risk patients [9].

\section{Radiation Therapy}

Radiation therapy has potential long-term cardiovascular complications. Radiation therapy itself can cause myocardial fibrosis, coronary arteriopathy, valvular dysfunction, constrictive pericarditis, and, as a result, diastolic dysfunction. The major coronary arteries are often directly exposed to radiation, and this can cause accelerated atherosclerosis with plaque rupture, thrombosis, as well as the potential to cause coronary spasm [10]. This effect has been seen in those treated for Hodgkin's lymphoma - these patients have an almost 6-fold increased risk of cardiovascular disease compared to the general population. This usually manifests clinically $15-20$ years after therapy with up to $50 \%$ of patients having some form of cardiovascular disease 40 years after radiation [8]. To 
add to this, these patients are often treated with chemotherapy regimens including anthracyclines, which is associated with often irreversible cardiac damage. Indeed, anthracyclines were the first drugs noted for cardiotoxicity already in 1967. Due to the identification of anthracycline cardiotoxicity, strategies for preventing anthracycline-associated cardiotoxicity have been developed. These include dosage reductions, limited cumulative dose, liposomal doxorubicin use, and continuous infusions, and some regimens have opted to avoid anthracyclines altogether in those at high risk for cardiotoxicity. Furthermore, dexrazoxane, a chelating agent, has been found to protect tissue from anthracycline cytotoxicity and is indicated for use to prevent doxorubicin cardiomyopathy [11]. These measures have been largely successful in minimizing the cardiovascular burden in these patients.

\section{Cardiovascular Prevention and Screening}

An initial baseline risk assessment is vital in all patients; this includes identifying traditional cardiovascular risk factors, treating comorbidities, performing an initial electrocardiogram with emphasis on the corrected QT interval, as well as performing a baseline echocardiogram (echo).

There is an increasing focus on using diagnostic tests for early, preclinical, detection of cardiotoxicity. This would help to identify those that could benefit from cardioprotective treatments, as well as enable early adjustment of cancer therapy before further cardiac injury is done. The most widely investigated tests have been the use of biomarkers and cardiac imaging.

The aim of appropriate imaging is not only to assess cardiac structure and function but also to identify subtle signs of early cardiac injury. There are many modalities available, including echocardiography, nuclear cardiac imaging and magnetic resonance imaging (MRI). Echocardiography is often used as the initial imaging modality as it is widely available and has no radiation exposure. And unlike most aspects in cardio-hemato-oncology, there is a consensus on the echo-guided definition of cancer therapeutics-related cardiac dysfunction (CTRCD). This is defined as a decrease of left ventricular ejection fraction (LVEF) of $10 \%$ to a value below the lower limit of normal $(<50 \%)$ [12]. This definition assists clinicians to identify and estimate CTRCD in a reproducible manner. Another measurement that can be done on echo is global systolic longitudinal strain which has been reported to be a predictor of early LV dysfunction in patients undergoing cancer therapy [13]. We expect that in the future, this will become a vital part of all patient assessments to prevent CTRCD.

Cardiac MRI is another useful imaging modality that can assess cardiac structure and function and is reproducible. It can also evaluate the pericardium in patients after radiation therapy, characterize myocardial tissue, such as areas of scarring, and assess for cardiac infiltrative diseases, such as amyloidosis.

Furthermore, biomarkers such as troponin and natriuretic peptides have been shown to be helpful in identifying those at high risk of cardiotoxicity as well as detecting early myocardial dysfunction. However, their use in guiding therapy and outcomes is yet to be seen.

\section{Cardioprotective Drugs?}

In 2016, the European Society of Cardiology published a position paper on cancer treatment and cardiovascular toxicity that has helped to guide clinical decision-making [10]. It recommends adding cardioprotective drugs, such as angiotensin-converting enzyme inhibitors (or angiotensin II receptor blockers), in combination with $\beta$ blockers if CTRCD is detected.

Some advocate adding cardioprotective drugs, such as angiotensin-converting enzyme inhibitors/angiotensin II receptor blockers, $\beta$-blockers, aspirin, and statins, as prophylactic therapy in patients undergoing cancer treatment. In the OVERCOME trial, patients receiving enalapril and carvedilol did not experience a reduction in LVEF at 6 months as compared to a reduction in LVEF in those that did not receive these drugs, and this translated into a lower incidence of major adverse cardiac events [14]. This data is promising, and we feel that without contraindications, prophylactic cardioprotective therapy should be encouraged.

Furthermore, strategies to diminish the complications of therapies have been adopted (such as those described above in anthracycline use). These include limiting the cumulative dose of cardiotoxic drugs and using radiation shields and inspiration breath-hold techniques during radiotherapy, to name but a few. Lifestyle changes, healthy nutrition, and exercise should also be encouraged. Educating patients on the potential side effects is vital, as well as vigilant patient follow-up and monitoring. This monitoring should not only be clinical but also via biomarkers and imaging, which can help to detect myocardial dysfunction before it becomes evident clinically. 
Table 1. Cardio-hemato-oncology: present and future

\begin{tabular}{|c|c|c|}
\hline The clinical problem & Therapeutic strategy & Remaining challenge \\
\hline $\begin{array}{l}\text { Cancer patients are at high risk for cardiovascular } \\
\text { disease due to their baseline cardiovascular risk factors, } \\
\text { the cancer itself, and potentially cardiotoxic therapies. } \\
\text { These therapies include agents with a dose-dependent } \\
\text { potentially irreversible cardiotoxicity (e.g., } \\
\text { anthracyclines), drugs with potential vascular toxicity } \\
\text { (e.g., tyrosine kinase inhibitors and immunomodulatory } \\
\text { agents), as well as those with potential long-term } \\
\text { cardiovascular complications (e.g., radiation therapy). } \\
\text { The balance between acquiring a hematological/ } \\
\text { oncological response and cardiovascular morbidity and } \\
\text { mortality requires expertise to optimize patient } \\
\text { outcomes. }\end{array}$ & $\begin{array}{l}\text { The field of cardio-hemato-oncology } \\
\text { encompasses a multidisciplinary service } \\
\text { geared towards hemato-oncology } \\
\text { patients that are at risk/suffer from } \\
\text { cardiovascular disease before, during, } \\
\text { and after cancer treatment. } \\
\text { This service should include appropriate } \\
\text { baseline assessments of patients, } \\
\text { screening for potential cardiotoxicity } \\
\text { during treatment, the addition of } \\
\text { cardioprotective drugs, and adequate } \\
\text { patient monitoring and follow-up. }\end{array}$ & $\begin{array}{l}\text { This field is in its infancy } \\
\text { with few specialists and is } \\
\text { often underutilized. } \\
\text { With the fast rate of the } \\
\text { development of new } \\
\text { therapies, some cardiotoxic } \\
\text { side effects are under- } \\
\text { diagnosed, leading to cardiac } \\
\text { morbidity and mortality. } \\
\text { Standardized diagnostics and } \\
\text { treatment guidelines need to } \\
\text { be established and } \\
\text { implemented. }\end{array}$ \\
\hline
\end{tabular}

\section{What Lies Ahead?}

New therapies are constantly emerging with their own side effect profiles. One such example is the increasing use of immune checkpoint inhibitors and the increasing reporting of often-fatal myocarditis associated with these drugs $[15,16]$. How do we screen for those at risk for myocarditis? Should checkpoint inhibitor therapy be interrupted once myocarditis is detected? Can cardioprotective drugs provide primary prevention?

These dilemmas highlight the complexity of these patients - the balancing between the high risk of mortality due to the hemato-oncological diagnosis and the cardiovascular morbidity in those that survive (Table 1). The field of cardio-hemato-oncology aims to provide a holistic multidisciplinary approach to the management of these patients before, during, and especially after initial therapy and to individualize patient therapy.
It is important to value the patient's perspective during this fragile time. These patients often have many unanswered questions, uncertainties, and fear. The value of having a team specialized in the intricacies of this field can help patients get more informed about the risks and benefits of therapy and empower patients in the decisionmaking process. We feel that a treatment approach as a holistic team can translate into improved overall satisfaction, a sense of optimized medical care, and ultimately improved patient outcomes.

\section{Statement of Ethics}

The authors have no ethical conflicts to disclose.

\section{Disclosure Statement}

The authors have no conflicts of interest to declare.

\section{References}

1 Tan C, Tasaka H, Yu KP, Murphy ML, Karnofsky DA. Daunomycin, an antitumor antibiotic, in the treatment of neoplastic disease. Clinical evaluation with special reference to childhood leukemia. Cancer. 1967 Mar;20(3): 333-53.

2 Gujral DM, Lloyd G, Bhattacharyya S. Provision and Clinical Utility of Cardio-Oncology Services for Detection of Cardiac Toxicity in Cancer Patients. J Am Coll Cardiol. 2016 Mar; 67(12):1499-500.
3 Ewer MS, Ewer SM. Cardiotoxicity of anticancer treatments: what the cardiologist needs to know. Nat Rev Cardiol. 2010 Oct; 7(10):564-75.

4 Valent P, Hadzijusufovic E, Schernthaner GH, Wolf D, Rea D, le Coutre P. Vascular safety issues in CML patients treated with BCR/ABL1 kinase inhibitors. Blood. 2015 Feb;125(6):901-6. 
5 Cortes JE, Kim DW, Pinilla-Ibarz J, le Coutre P, Paquette R, Chuah C, et al.; PACE Investigators. A phase 2 trial of ponatinib in Philadelphia chromosome-positive leukemias. $\mathrm{N}$ Engl J Med. 2013 Nov;369(19):1783-96.

6 U.S. Food and Drug Administration. FDA Drug Safety Communication: FDA requires multiple new safety measures for leukemia drug Iclusig; company expected to resume marketing. Center for Drug Evaluation and Research; 2014 [cited 2018 Jul 28]. Available from: https://www.fda.gov/Drugs/DrugSafety/ucm379554.htm.

7 Caocci G, Mulas O, Annunziata M, Luciano L, Bonifacio M, Orlandi EM, et al. Cardiovascular toxicity in patients with chronic myeloid leukemia treated with second-generation tyrosine kinase inhibitors in the real-life practice: identification of risk factors and the role of prophylaxis. Am J Hematol. 2018 Jul; 93(7):E159-61.

8 Palumbo A, Rajkumar SV, Dimopoulos MA, Richardson PG, San Miguel J, Barlogie B, et al. Prevention of thalidomide- and lenalidomide-associated thrombosis in myeloma. Leukemia. 2008 Feb;22(2):414-23.
9 Palumbo A, Rajkumar SV, San Miguel JF, Larocca A, Niesvizky R, Morgan G, et al. International Myeloma Working Group consensus statement for the management, treatment, and supportive care of patients with myeloma not eligible for standard autologous stem-cell transplantation. J Clin Oncol. 2014 Feb;32(6):587-600

10 Zamorano JL, Lancellotti P, Rodriguez Muñoz D, Aboyans V, Asteggiano R, Galderisi M, et al.; ESC Scientific Document Group. 2016 ESC Position Paper on cancer treatments and cardiovascular toxicity developed under the auspices of the ESC Committee for Practice Guidelines: the Task Force for cancer treatments and cardiovascular toxicity of the European Society of Cardiology (ESC). Eur Heart J. 2016 Sep;37(36):2768-801.

11 van Dalen EC, Caron HN, Dickinson HO, Kremer LC. Cardioprotective interventions for cancer patients receiving anthracyclines. Cochrane Database Syst Rev. 2011 Jun;(6): CD003917.

12 Plana JC, Galderisi M, Barac A, Ewer MS, Ky B, Scherrer-Crosbie M, et al. Expert consensus for multimodality imaging evaluation of adult patients during and after cancer therapy: a report from the American Society of Echocardiography and the European Association of Cardiovascular Imaging. Eur Heart J Cardiovasc Imaging. 2014 Oct;15(10):106393.
13 Sawaya H, Sebag IA, Plana JC, Januzzi JL, Ky $\mathrm{B}$, Cohen V, et al. Early detection and prediction of cardiotoxicity in chemotherapy-treated patients. Am J Cardiol. 2011 May;107(9): 1375-80.

14 Bosch X, Rovira M, Sitges $M$, Domènech $A$, Ortiz-Pérez JT, de Caralt TM, et al. Enalapril and carvedilol for preventing chemotherapyinduced left ventricular systolic dysfunction in patients with malignant hemopathies: the OVERCOME trial (preventiOn of left Ventricular dysfunction with Enalapril and caRvedilol in patients submitted to intensive ChemOtherapy for the treatment of Malignant hEmopathies). J Am Coll Cardiol. 2013 Jun;61(23):2355-62.

15 Mir H, Alhussein M, Alrashidi S, Alzayer H, Alshatti A, Valettas N, et al. Cardiac Complications Associated With Checkpoint Inhibition: A Systematic Review of the Literature in an Important Emerging Area. Can J Cardiol. 2018 Aug;34(8):1059-68.

16 Johnson DB, Balko JM, Compton ML Chalkias S, Gorham J, Xu Y, et al. Fulminant Myocarditis with Combination Immune Checkpoint Blockade. N Engl J Med. 2016 Nov;375(18):1749-55. 